\title{
CUA-AUA International Fellows Program: San Diego 2016
}

\author{
Edmund C.P. Chedgy, MBBS, MSc, FRCS (Urol) \\ Department of Urologic Sciences, University of British Columbia, Vancouver, BC, Canada
}

Cite as: Can Urol Assoc J 2016;10(7-8):E279. http://dx.doi.org/10.5489/cuaj.4079

Published online August 16, 2016

$\mathrm{H}$ aving received an email from the Canadian Urological Association (CUA) informing me that the application window for this prestigious award had been extended, I jumped at the chance to apply. I was lucky enough to be selected and subsequently made arrangements to head down to San Diego, CA from Vancouver, BC, where I am currently an uro-oncology fellow. This program represents a unique opportunity for trainees in the latter stages of their training to visit the America Urological Association (AUA) meeting in a structured and supported fashion. In total, there were seven lucky candidates. It is certainly not something that is available back in the U.K.

At the pre-conference WebEx briefing, our distinguished mentors discussed the outline and goals of the program. Advice on maximizing our learning opportunities and navigating the conference efficiently was also given. The advice proved invaluable, given the sheer enormity of the AUA.

We were given the task of selecting and reviewing two abstracts: one from a recommended list of prostate cancer posters, the other a free choice. We were then to report on these, in a structured presentation, at the review dinner held on the last night of the conference.

The hot topics in uro-oncology centred on prostate multiparametric magnetic resonance imaging (MRI) and the somewhat controversial high-intensity focused ultrasound (HIFU). Both generated plenty of discussion among conference delegates and in the Twitter-sphere. There were also new AUA guidelines on non-muscle-invasive bladder cancer (NMIBC) and the surgical management of stones, with an update in castrate-resistant prostate cancer (CRPC).

Given that my current research is focused on prostate cancer, I spent most of my time in prostate cancer-based moderated poster sessions. In particular, I chose to review an abstract evaluating orthotopic xenografts using spheroid cell cultures in a preclinical prostate cancer model. ${ }^{1}$ The authors were able to demonstrate successful tumour growth using a number of modalities, including ultrasound and MRI, with measurable changes in mouse plasma prostate-specific antigen (PSA). The successful generation of a preclinical xenograft model in this context opens potential new pathways in the development of patient-derived xenograft (PDX) models in prostate cancer. While currently, these techniques are likely too expensive to bring into routine clinical practice, their potential to allow clinicians to make treatment decisions based on the response of PDX models to therapy brings the prospect of true precision oncology ever closer to reality. Furthermore, they have the potential to garner insight into the effectiveness of novel therapies for prostate cancer that will have alternate targets than the androgen receptor (AR). While the abstract demonstrated important potential for future advances, significantly more work will need to be done to make these techniques common practice.

The highlight of the International Fellows Program was the dinner and presentation at the close of the conference. This event offered an opportunity to hear in-depth reports and critique of a number of abstracts and presentations ranging from the update in stone guidelines to robotic caval thrombectomy for renal tumours. The ensuing discussions were lively and interactive, and we were fortunate to draw upon the knowledge of our expert mentors to facilitate these.

While the AUA this year was both enjoyable and informative, there was a feeling that there were no immediate practice-changing updates, but rather a wealth of potential future advances in various stages of development - much like the abstract I chose to review.

Competing interests: The author reports no competing personal or financial interests.

\section{Reference}

1. Saar $M$, Linxweiler J, Valta $M$, et al. Mp62-12 orthotopic xenografts using LuCaP136 spheroid cultures provide a versatile predinical model of prostate cancer. J Urol 2016; 195:e816-e7. http://dx.doi. org/10.1016/i.juro.2016.02.920

Correspondence: Dr. Edmund C.P. Chedgy, Department of Urologic Sciences, University of British Columbia, Vancouver, BC, Canada; echedgy@prostatecentre.com 